\title{
ON THE TREATMENT OF EPIDIDYMITIS BY OIL OF YELLOW SANDAL WOOD, AND ON THE MODE OF ACTION OF THAT OIL. -
}

\author{
BY ROBERT IVHARRY, II. D.
}

of loxvox.

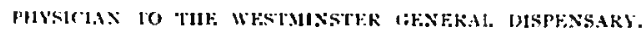

7 THERE is a general consensus of opinion that sandal oil influencc upon gonorrhosa and it is believed they act through the medium of the urine. Ricrod's observations have led him to the conclusion that copaiba acts in this way. He supposes that when taken into the system it becomes excreted by the urine in some mysteriously altered form, and during micturition comes into contact with the nucous membranc of the urethra, acting as an injection. The corrcctness of this view is open to question.

It is based upon three cases. In thesc he scems to have forgotten the "ceteris paribus" and compared the part of the urethra washed out repeatcdly every day by the copaiba charged urine with that part which was not washed out at all by either urine or simple water. (In those cases where the discharge has been allowed to remain in contact with the urethral surface the aggravation of the discase is notorious). He leaves unexplained the rccords of the unsatisfactory effects of using copaiba charged urinc as an injection. Also, he does not prove that if the copaiba is changed in the urine that it is not already similarly changed while in the blood, i. e., that the mysterious change in the copaiba takes place in the kidney and not in the liver or elsewhere. Finally, he docs not prove that if the copaiba undergoes the ncccssary mysterious change 
while yet in the blood it does not get as near to the seat of mischief while circulating through the capillaries of the urethral mucous menbrane as while passing with the urine through the uretlira. A better explanation of the facts observed con-. cerning the influence of copaiba is that it acts in two directions at once: in the first place, while circulating in the capillaries of the urethral nucous membrane and. in the second, while passing with the urine through the urethra. This is the way l believe copaibal acts, and this is the way 1 believe oil of sandal wood acts in checking gonorrhu:al.

Having a patient with a swelled testicle from gonorrbceit, and finding all sorts of ordinary measures were of little use, I cast about for some means of hastening recovery, and in a conversation with Mr. C. 13. Keetley he let fall the suggestion of sandal oil. This was accordingly tried, and with very excellent results. The patient improved rapidly and in a week was practically well, there being no tenderness on pressure and but little enlargement.

In the second case in which 1 tried it, it answered equally well. In this the patient had been under treatment for about three weeks before coming under iny care and was surprised and pleased with the apparent immediate improvement.

ln the third case the patient was first seen when the inflammation was acute and the testicle as large as one's fist. In ten day's he was up and about, wanting to begin training for a bicycle race.

In the fourth case an officer in the arny, having grown impatient with his tardy progress. came to me, and was, in his uwin opinion, well within seven days. Upon a subsequent uccasion he again suffered with swelled testicle, came to me and was put on the sandal oii, improved rapidly for three days, and then, wishing to avoid some military duty, applied to be put un the sick list. The army surgeon insisted upon treating him ur else refused to put him on the sick list, so the sandal oil was discontinued and other means substituted. He grew rapidly. worse and in al fortnight came to see me again, improved rapidly, and got well by taking the sandal oil and pouring the uther physie ouwn the sink.

These results, obtained in a trial undertaken without any pre- 
coneeived theory or prejudice, encouraged me to use the sandal oil in gonorrhoeal rheumatism, and this 1 have done in two cases, with the best results. In the first case 1 tried manythings for three wecks without causing the least improvement. Immediately upon giving sandal oil the improvement began and recovery rapidly became complete. In this case I had tried salieylate of soda in full doses without effeet.

In the seeond ease I gave the sandal oil immediately un the appearance of the rheumatism in the ankles, and all symptoms were gone in two days.

I have given sandal oil in several cases of subacute prostatitis and though these cases all did well and rapidly reeovered, other means were used in addition, and eases usually do well without sandal oil.

In one ease I attempted to prevent the formation of : 1 : orrhoeal bubo by giving sandal oil, but not successfully.

Such is my experience of sandal oil in the treatment of the complications of gonorrhoa. Though not sufficient to prove much, it has left a ven strong impression upon ny mind that it stands a long way aluead of any other remedy ior treating "swelled testiele" and gonorrhozal rheumatisn.

It may' be as well to add that in cases of long standing gonorrhoeal rheumatism it would be unreasonable to expect reeorery in a few days under sandal oil. The best results may be hoped for in eases where this treatment is begun very early: and in old long-standing eases better results may be hoped for in a treatment which includes sandal nil than in nne which excludes it. 